\title{
Psicología y Ley: "Una relación Particular". Concepto, desarrollo histórico y áreas de intervención de la Psicología Jurídica
}

\section{Psychology and Law: "A Particular Relationship". Concept, historic development and intervention areas of Legal Psychology}

\author{
Luis Cárcamo Montero ${ }^{1}$ \\ Universidad Complutense de Madrid
}

(Recepción: Marzo 2006 - Acepatción: Junio 2006)

\begin{abstract}
Es probable que en el futuro, esta última década sea considerada como un periodo clave en el desarrollo de la Psicología Jurídica en nuestro país. En 1995, se realizaba en Chile el Primer Congreso Iberoamericano de Psicología Jurídica. Diez años después, las reformas legales implementadas, la asociación y mayor coordinación de los profesionales del área, la realización de encuentros científicos nacionales e internacionales, publicaciones y la multiplicación de ofertas de formación, dan cuenta del momento de expansión que vive esta especialidad.

Considerando cste momento, el presente artículo se propone revisar el concepto de Psicología Jurídica, abordando su evolución histórica en países como España, Alemania y Estados Unidos. Posteriormente se describen sus diversos campos de aplicación (Menor y Familia, Penitenciaria, Policial, Victimología, Forense c Investigación y Docencia) contextualizando su quchacer en el escenario nacional.
\end{abstract}

Palabras clave: Psicologia, ley, psicología juridica.

It is probable that in the future, this last decade may be considered as a clue period in the development of Legal Psychology in our country. On 1995, was carried out in Chile the first Iberoamerican Congress of Legal Psychology. Ten years later, the legal reforms, the association and improvement in coordination among the arca professionals, the realization of national and international scientific meetings, publications and the multiplication of learning offers, talk about the moment of expansion that this speciality is living.

Considering this moment, the present article proposal is to review the concept of Legal Psychology, approaching to its historic revolution in countrics as Spain, Germany and the United States. Afterwards, the diverse fields of application are described (Child and Family, Penitentiary, Policial, Victimology, Forensics and Investigation and Teaching) contextualizing its application on a national scenario.

Keywords: Psychology, Law, Legal Psychology.

\section{Psicología y Ley}

Si bien el Derecho y la Psicología son disciplinas que comparten un mismo objeto de estudio, sus objetivos son diferentes: mientras el derecho busca regular la conducta humana, la psicología por su parte, se preocupa de su explicación (Kapardis, 1997). De esta manera, ambas según Sobral (1994) tienen su encuentro, en torno a los procesos que gobiernan la conducta ${ }^{2}$. Pese a lo anterior, ambas ciencias se han desarrollado de forma paralela y con un relativo olvido mutuo (Soria, 1998).

I Correspondencia a: Luis Cárcamo M. E mail: lkarkamo@gmail.com

2 Así, por ejemplo, cl Derecho Penal no tendría sentido, si no se presupone que la conducta del hombre se rige como lo predicen los principios conductistas.
A través del tiempo algunos autores han considerado a la Psicología como fundamento de la Ley positiva ${ }^{3}$ y de sus aplicaciones concretas (Monahan y Loftus, 1982; Haney, 1984), teniendo un papel fundamental tanto en su formulación racional, como en la aplicación y evaluación de sus resultados ${ }^{4}$.

3 En la visión positivista de la Ley, esta se considera como un sistema de normas que se distingue por su obligatoriedad, cayendo en el campo del deber ser, siendo éstas válidas aunque $\mathrm{cl}$ sujeto las viole.

4 Citado con frecuencia como un hecho emblemático por los psicólogos que defienden esta postura, es la sentencia del Tribunal Supremo de los Estados Unidos (1954), en la que se prohibió la educación discriminada entre negros y blancos, afirmando explícitamente que las ciencias sociales habían demostrado que la educación separada promovia la segregación. Los detractores de ésta, acusaron al tribunal de apartarse de su obligación de tomar decisiones puramente legales y de contaminarlas con consideraciones psicológicas y sociológicas. 
De este modo las relaciones entre Psicología y ley son antiguas, $y$ han pasado por diferentes estados y grados de aproximación, lo que ha dependido fundamentalmente del propio desarrollo interno de cada una de ellas. De acuerdo con Loh (1981), la historia de las relaciones entre ambas disciplinas mostraría tres principales tipos de acercamiento:

a) La necesidad de contar con los descubrimientos de la Psicología en la obtención de evidencias judiciales,

b) La calificación científica del psicólogo para emitir evaluaciones forenses, $y$

c) La influencia de los conocimientos psicológicos sobre el cambio legal.

En la actualidad se establece que la Psicología posee una mirada y un accionar que supera la auxiliaridad a la que fue históricamente convocada desde el ámbito jurídico por ciencias como la Medicina y la Psiquiatría, con prácticas que se prestaban a determinar aquello que era "anormal" para la sociedad. Según Sobral (1998), se requiere superar estos preceptos de corte positivista, posición más destacada a la hora de concebir sus posibles acciones.

En nuestro país esta disciplina ha tenido, hasta hace poco, escaso desarrollo y según Escaff (2000), sólo desde hace algunos años se ha visto una creciente participación de psicólogos en el ámbito de justicia. Incremento que se ha visto impulsado entre otras cosas por la modificación del sistema procesal penal y la ley que crea los Tribunales de familia, los que han implicado la creación de nuevas plazas para psicólogos dentro de la administración de justicia.

Cabe destacar la histórica participación de estos profesionales en Gendarmería de Chile, al interior del Centro de atención a víctimas de atentados sexuales (C.A.V.A.S.) dependiente de la Policía de Investigaciones, así como en instituciones de la red del Servicio Nacional de Menores (SENAME).

\section{Hacia una definición de Psicología Jurídica}

Existen diversas perspectivas sobre la Psicología Jurídica, las que a su vez determinan distintas definiciones de ésta. En primer lugar cabe señalar que el término Psicología Jurídica tiene su origen en la obra de Emilio Mira y López en 1932, quien define a la Psicología Jurídica como un campo situado entre la Psicología y la Psiquiatría, cuyo objetivo es prevenir y tratar a los delincuentes, apuntando, no obstante, a otras áreas tales como el testimonio, la evidencia delictiva, la comprensión del delito, la motivación psicológica del mismo, la confección de informes forenses y la reforma moral del delincuente.

Muñoz Sabaté (1975) presenta una triple visión de la Psicología Jurídica ${ }^{5}$, inclinándose por la idea de una Psicología aplicada y afirmando que ésta hace referencia a la aplicación de los métodos y conocimientos psicológicos tanto a la ciencia jurídica como a la práctica del Derecho. En su opinión, la Psicología Jurídica sólo se entiende en y para el Derecho, y aun en este caso sólo en lo que se refiere a la interpretación de casos jurídicos concretos, en tanto pueda aplicar sus técnicas y conocimientos a la prueba de determinados sucesos. Asimismo, la Psicología Jurídica debe atenerse a la norma jurídica, sin valorar si es justa o no, ni pretender argumentar sobre sus fines, tareas que en su opinión se inscribirían más bien dentro de una Psicología General.

En 1978, Teixido afirma que el objeto de estudio de la Psicología Jurídica es el análisis y la descripción de aquellas conductas y motivaciones que traspasan la propia individualidad (objeto de la Psicología pura) para producir efectos jurídicos.

Garrido (1982) define a la Psicología Jurídica como una unión entre la Psicología General y la Criminología, en la que se tratan de aplicar los conocimientos y los métodos de la Psicología a la resolución de los problemas del Derecho.

\footnotetext{
5 Afirma que lo que se entiende por ella cambia, sea que se tome como Psicologia del Derecho, en la que se partiria de la idea de que casi todo el Derecho está lleno de componentes psicológicos, por lo que requeriria a la Psicologia para su funcionamicnto; Psicología en el Derecho, la Psicología es un campo al que el jurista pucde acudir en busca de asesoramiento para la definición normativa, ya que tanto la formulación como la aplicación de las normas juridicas son áreas que se ven afectadas por una gran cantidad de variables tanto ambientales como propias de los sujetos, partiendo de la base de que el conocimiento de las mismas favorece el logro de la máxima eficiencia juridica o Psicologia para el Derecho, en la cual la Psicologia actuaría como auxiliar del Derecho cuando éste necesita información sobre sus fines, sus estrategias o cuando necesita interpretar hechos relativos a casos especificos.
} 
En 1989, Garzón nos da una definición en la que no aparece la idea de aplicación de los conocimientos psicológicos. En su opinión, la Psicología Jurídica sería una ciencia que trata de describir y explicar los supuestos psicológicos del poder judicial, los procesos cognitivos de la justicia (representaciones, creencias, actitudes) y los procesos y fenómenos psicológicos de los diferentes actores judiciales que concretizan los distintos marcos ideológicos de los sistemas judiciales.

En opinión de Clemente (1997) la Psicología Jurídica es una aplicación de la Psicología Social, y coincide con Soria (1998) en considerarla como el estudio del comportamiento de las personas y de los grupos, en cuanto éstos tienen la necesidad de desenvolverse dentro de ambientes regulados jurídicamente, así como de la evolución de dichas regulaciones jurídicas o leyes, y los procesos psicosociales que las guían o facilitan.

Es posible constatar que no existe consenso en su definición, sus tareas y ámbitos de intervención. Cuestionándose incluso la conveniencia de usar el concepto de "Psicología Jurídica" por considerarlo algunos un término demasiado amplio, acusando cierta ambición en sus objetivos, que se verían limitados por el temor a una psicologización excesiva del derecho. Para los representantes de esta postura resulta preferible utilizar el término "Psicología Forense", coincidiendo con los países de habla inglesa. Este hace alusión a lo ocurrido en la sala o "foro", por lo que a su vez resulta restrictivo, ya que se refiere fundamentalmente a la realización de informes periciales.

La Asociación Chilena de Psicología Jurídica, propone una visión integral, concibiendo el trabajo del Psicólogo en esta área, en términos transdisciplinarios, el que abarca distintos ámbitos y niveles de intervención, en el que se incluyen las labores de asesoramiento, formación, intervención, evaluación y diagnóstico, prevención social y mediación, entre otros, destacando el papel que desempeña el psicólogo forense en los procesos judiciales, además del trabajo teórico y de investigación.

\section{Desarrollo histórico de la Psicología Jurídica}

Es posible situar el acercamiento entre Psicología y Derecho a principios del siglo XX, el que se produce para intentar responder a ciertas necesidades jurídicas, como la de asegurar una mayor fiabilidad de las declaraciones judiciales, convirtiéndose según Bajet (2003), en una de las especialidades más antiguas de la Psicología aplicada.

En Alemania, la Psicología Jurídica es una de las materias con la que parte la Psicología, destacándose por su tradición en investigación y práctica. A comienzos del siglo XX, hacen el papel de peritos judiciales los representantes de la materia, realizándose intensas investigaciones en el tema del testimonio (Fabian, 2000). En España en tanto, surgen los primeros estudios empíricos, orientados a temas como la Psicología del testimonio y la criminología (Soria, 1998).

En Estados Unidos, Munstberg (1907) desarrolla las bases del testimonio, no ocurriendo mucho más sino hasta 1928, año en que Hutchins y Slesinger comienzan una serie de artículos en el mismo tema, en un período que dura cerca de diez años.

Posteriormente, en las décadas de los años treinta y cuarenta, se observa un énfasis en temáticas relacionadas con el ámbito penal y criminológico. En España, se distinguen dos escuelas importantes: la de Madrid, centrada en la Psiquiatría Forense, con una preocupación hacia el desarrollo de una actitud preventiva frente a las personalidades peligrosas, distinguiendo las bases biológicas, psicológicas y sociales de la delincuencia, y Barcelona con una visión holística y personalista de la Psicología. Emilio Mira y López, uno de sus principales exponentes, publicó en el año 1932 el Manual de Psicología Jurídica (Soria, 1998).

Un año antes (1931), Burtt publica en Estados Unidos la obra titulada "Legal Psychology"; esta se mueve desde principios generales de la Psicología, a las aplicaciones de ésta en la administración de justicia.

En adelante, y hasta la década de los cuarenta las relaciones entre Psicología y Ley experimentan pequeños progresos (Tapp, 1976), posiblemente afectadas por los conflictos internacionales.

Alrededor de las décadas de los años cincuenta y sesenta, se produce la profesionalización del ámbito de la Psicología Jurídica, afianzándose ésta como disciplina independiente y con existencia propia, ampliando su campo de acción en diversas temáticas, tanto civiles como penales. 
Posteriormente, en los años setenta y ochenta, la incorporación de la Psicología al ámbito legal se amplía aún más, incluyendo la confección de informes, la toma de declaraciones en diversos casos, tanto civiles como penales, la asesoría a la labor policial y penitenciaria, además de un notable aumento de la investigación.

En Estados Unidos, durante los setenta, surge la preocupación por encontrar los factores que determinan la conducta, aquellos sobre los que yacen el orden social y legal, produciéndose un renovado y mutuo interés por parte de ambas ciencias. Los expertos comenzaron a ser llamados para atestiguar acerca de la fiabilidad de los testigos presenciales. Junto con esto surgen controversias respecto de la influencia de la Psicología en las decisiones judiciales, lo que aumentó el interés en la investigación en el área (Blair, 1972).

En España se observa un período de efervescencia, renacer en el que confluyeron factores como la primera promoción de psicólogos españoles en 1971, la aparición del primer "Anuario de Sociología y Psicología Jurídica" dirigido por Muñoz Sabaté en 1974, y la aparición del Manual "Introducción a la Psicología Jurídica” de Muñoz Sabaté, Bayés y Munné en 1980. En la década de los ochenta se produce la incorporación del psicólogo en las instituciones penitenciarias, se forma el Colegio Profesional de Psicólogos en 1980, mientras que al año siguiente aparece la Ley de Divorcio, que llevó a la creación de los Juzgados de Familia y la inclusión del psicólogo como técnico de consulta en temáticas legales (Soria, 1998).

En esta misma época surgen las asociaciones que agrupan a los profesionales de esta área. En 1970, la American Psychological Asociation, APA, define el rol del psicólogo jurídico y se constituye la Sociedad Americana de Psicología y Ley ${ }^{6}$. Dicha organización, tiene por objetivo buscar el aumento de las contribuciones de la Psicología a la comprensión de la ley en las instituciones legales, a partir de la realización de investigaciones básicas y aplicadas, promoviendo también la formación de psicólogos en estas materias e informar a la comunidad acerca de sus actividades.

La Federación Alemana de Asociaciones de psicólogos, funda en 1984 la Agrupación de

6. División $41^{\circ} \mathrm{del}$ organismo.
Psicología Jurídica, que cada dos años lleva a cabo una jornada de trabajo en la que se abordan diversos temas clave. Por otra parte, a principios de la década de los setenta, el Colegio de Psicólogos alemanes fundó en su seno la Sección de Psicología Forense, cambiando su nombre en 1992 a Sección de Psicología Jurídica (Fabian, 2000).

En 1987 se creó la primera Comisión de Psicología Jurídica al interior del Colegio de Psicólogos de España y en 1988 se creó, "oficialmente", la figura del "psicólogo jurídico".

La década de los noventa se caracteriza por un aumento en la investigación, congresos y publicación de libros y artículos especializados. Investigación que en Alemania está centrada principalmente en la psicología del testimonio y criminal. Este período es considerado por Soria (1998) como "La edad de Oro de la Psicología Jurídica Española”. Significando el establecimiento de una identidad, consolidando y ampliando diversas áreas de intervención.

En cuanto a la formación y perfeccionamiento, en Alemania, los estudios adicionales en el área tienen un mínimo de tres años de duración, basándose principalmente en la participación en seminarios, la supervisión constante por parte de un equipo especialista y la práctica permanente en la realización de exámenes psicológico-forenses prácticos (Fabian, 2000).

En Estados Unidos existen hoy en día, diversos programas de entrenamiento en el área de la Psicología y Ley, encontrándose en el sitio web de la división correspondiente de la APA un total de diecinueve programas de doctorado en la materia. Como una manera de garantizar la calidad y preparación de los psicólogos que se desempeñan en el ámbito forense, la Asociación Americana de Psicología Forense ofrece un diploma reconocido por la APA, el que certifica que dicha organización ha examinado y aceptado al postulante y lo califica para desempeñarse en el área y en las tareas propias de la práctica forense.

\section{Ámbitos de estudio e intervención de la Psicología Jurídica}

La clasificación de los distintos ámbitos de la Psicología Jurídica no es unánime, debido a las peculiaridades culturales, científicas y legislativas de cada país. Clemente (1997) identifica 
quince temáticas susceptibles de ser estudiadas por la Psicología Jurídica, por otra parte Soria (1998) señalará que esta es posible dividirla en doce grandes áreas. Es claro que éstas varian según se van consolidando sus tareas específicas, debiendo cada uno establecer sus límites y estados fronterizos con las otras áreas con las cuales colabora de manera interdisciplinaria.

Escaff (2000) resume en seis, los principales ámbitos en los que se desempeñan los profesionales de la Psicología en su relación con lo judicial. Esta clasificación es la que utilizamos a continuación:

\section{a. Psicología Juridica del Menor y la Familia}

La preocupación por el menor, en casi todos los países, es objeto de una política integral, desarrollada desde diferentes ámbitos: educativo, sanitario, servicios sociales, y judicial, entre otros. Es desde este último, que tiene sentido entonces hablar de Psicología Jurídica del Menor.

En esta área, habitualmente el psicólogo forma parte de equipos multidisciplinarios, ya sea en instituciones que trabajan en educación y protección de menores o en consejos técnicos. Estos trabajan en colaboración directa con el juez, participando en la evaluación de niños o adolescentes vulnerados gravemente en sus derechos, o que hayan realizado hechos susceptibles de ser tipificados como delitos. Esta evaluación ha de contemplar la situación psicológica, educativa y familiar del menor, además de otras circunstancias que resulten relevantes, con el objetivo de emitir un informe y asesorar al juez o Fiscal acerca de la situación del niño o adolescente infractor, las posibilidades para su reeducación y tratamiento, así como proponer medidas socio-educativas más convenientes.

Destaca en esta área el trabajo realizado por los psicólogos que se desempeñan en la red asistencial del Servicio Nacional de Menores (SENAME), tanto en los centros de administración directa como los colaboradores privados, quienes realizan evaluaciones diagnósticas y propuestas de tratamiento, elaboración, implementación y seguimiento del proceso.

En cuanto al ámbito familiar, cabe destacar la entrada en vigor en noviembre del 2004 de la nueva Ley de Matrimonio civil No 19.947 y la Ley 19.968 que crea los Tribunales de familia, que comenzaron a funcionaron en octubre del año pasado. Estos tribunales cuentan con con- sejos técnicos en los que participan profesionales del área de la Psicología, con el objeto de asesorar al Juez en acciones de separación, nulidad y divorcio, procesos de adopción, casos de violencia intrafamiliar y maltrato infantil? filiación, tuición y patria potestad, alimentos, visitas, autorización para salida del país, medidas de protección, separación de bienes, declaración de interdicción, e infracciones penales cometidas por menores, entre otros.

Ambas leyes contemplan instancias de mediación familiar, la que proporciona una forma racional de resolver aquellos conflictos que tienen difícil solución o representan un costo elevado para los ciudadanos y para la sociedad. En ésta, la solución no viene dada del exterior, sino que la realizan las propias partes en conflicto con la ayuda de un tercero imparcial, el mediador, quien deberá favorecer la comunicación entre las partes y la consolidación de los acuerdos. Se espera que este sistema ayude a descongestionar los juzgados especialmente en materias de tuición, alimentos y disputa de visitas.

\section{b. Psicología Penintenciaria}

El psicólogo penitenciario forma parte de uno de los últimos eslabones del sistema de justicia penal. En nuestro país, su labor se lleva a cabo en el contexto de Gendarmería de Chile, institución dependiente del Ministerio de Justicia, cuya misión principal es "atender, vigilar y asistir a las personas que por resolución de las autoridades competentes, fueron detenidas, privadas de libertad o con libertad restringida, contribuyendo a su reinserción social"s.

Dada la complejidad del fenómeno criminógeno, el psicólogo penitenciario desarrolla su trabajo en contacto permanente con otros profesionales, entre los que podemos encontrar trabajadores sociales, educadores, terapeutas ocupacionales y sociólogos entre otros, desempeñando su trabajo en distintos organismos del sistema penitenciario, aunque fundamentalmente dentro de los denominados consejos técnicos, tanto en el subsistema cerrado como abierto.

También participa en los departamentos de Readaptación Social y de Tratamiento en el

\footnotetext{
7 Ley $\mathrm{N}^{\circ} 19.324$ sobre Maltrato de Menores y Ley $\mathrm{N}^{\circ}$ 19.325 sobre Violencia Intrafamiliar.

8 Decreto de Ley No 2.859 .
} 
medio libre, ambos encargados de asesorar a la Dirección Técnica Nacional en materias relativas a la asistencia y reinserción social de las personas que cumplen condena en los distintos subsistemas (cerrado, semiabierto y abierto), además de diseñar y proponer políticas tendientes a la rehabilitación de los internos.

Las funciones principales del profesional de la psicología en esta área implican la evaluación: a través de entrevistas, pruebas psicodiagnósticas, observación del comportamiento, examen de documentos e informes de otros profesionales, se realiza una evaluación integral de la personalidad del interno, para luego elaborar el correspondiente informe psicosocial que será remitido al órgano superior o a la autoridad judicial, que lo considerará a la hora de conceder algún beneficio intrapenitenciario o en la derivación al programa de tratamiento más apropiado.

Tratamiento: a través del diseño e implementación de programas y talleres, así como de la intervención terapéutica y acompañamiento de los internos, como delegado de libertad vigilada. Tratamiento que ha de estar basado en el estudio de la personalidad del individuo, siendo individualizado, complejo y programado. Cabe señalar que el tratamiento es tarea central y elemento transversal de la pena, con el objetivo de la posterior reinserción del interno, por lo que todas las variables, desde la clasificación y segmentación inicial, hasta las condiciones del medio, han de tender a este objetivo.

De acuerdo a Retamal (2002) es posible reconocer una polaridad existente en las funciones de evaluación y tratamiento, enmarcadas dentro del campo de la reinserción. Ya que en la medida que la evaluación y el diagnóstico destinado al otorgamiento de beneficios intra-penitenciarios se constituye en la función privilegiada del Psicólogo, se minimiza el impacto de las tareas que tienen como fin la reinserción social, que se ven generalmente postergadas.

Otras tareas podrían incluir la participación en los órganos colegiados, formación del personal de la institución e investigación.

El ámbito penitenciario ha sido históricamente uno de los con mayor participación de psicólogos en el país, cuyo número se ha incrementado significativamente en los últimos años, alcanzando a un número de 163 a nivel nacional en el año 2004, 77 de los cuales se desem- peñan en el sistema cerrado, 69 en el sistema abierto y 17 en funciones a nivel central de la institución. ${ }^{9}$ Importante ha sido en este sentido la modificación en el reglamento que rige los establecimientos penitenciarios ${ }^{10}$ en el año 1998, que dio una mayor importancia al informe psicológico en la evaluación de los avances del interno en sus niveles de reinserción. Esto implicó un mayor número de psicólogos en los penales, los que se desempeñan dentro de los denominados equipos técnicos de cada establecimiento.

Varios autores (Clemente, 1997; Ruiz y Páez, 2001) están de acuerdo en las importantes contribuciones que puede realizar la Psicología Organizacional a esta área, su aplicación en aspectos como la selección y capacitación de recursos humanos, la identificación e intervención sobre variables que influyen en el estrés laboral y en la satisfacción en el trabajo, así como en temas como cultura y clima organizacional. Con el objetivo de mejorar la gestión de las unidades penales, incidiendo en la calidad de vida de empleados e internos.

\section{c. Psicologia Policial}

Por lo general, las principales tareas de los psicólogos que se desempeñan dentro de los cuerpos de seguridad y las policías, dicen relación con temas como formación del personal, selección, organización y relaciones con la comunidad. Funciones en general clásicas de la Psicología Organizacional, aunque aplicadas al medio policial.

Sin embargo, a estas tareas tradicionales de la Psicología Policial, se están incorporando actualmente otras más complejas, como son la formación especializada de mandos intermedios y oficiales, así como de grupos especializados (unidades de menores, delitos sexuales, intervención en crisis, etc.). Además de la colaboración en la investigación policial través de la elaboración de perfiles criminales.

\footnotetext{
- Datos presentados por la Comisión de Psicología Penitenciaria de la Asociación Chilena de Psicología Jurídica en el Primer Encuentro Nacional de Psicología Jurídica, agosto de 2004.

${ }^{10}$ Decreto supremo No 518, promulgado el 21 de agosto de
} 1998. 


\section{d. Psicología de la Víctima}

Diversos autores (Soria, 1993; Esbec, 2000; Escaff 2000) coinciden al considerar que la víctima del delito fue por mucho tiempo la gran olvidada del proceso penal.

La victimología ha sido definida como "el estudio científico de las víctimas, especialmente de las víctimas del delito" (Jerusalem, Israel, 1973). Algunos la consideran una rama de la criminología, mientras que para otros, constituye una disciplina específica que recibe aportes de varias ciencias como la psicología, sociología, medicina, etc.

La criminología, luego de la segunda mitad del siglo XX intentando buscar nuevas explicaciones al fenómeno criminal, centra su atención ya no sólo en el delincuente, sino en su interacción con la víctima (Esbec, 2000).

Según Soria (1993) son reconocidos como precursores de esta área, Mendelsohn, criminólogo israelí que introdujo el término victimología en 1947, durante una conferencia celebrada en Bucarest, y Von Heting, con la publicación de "The criminal and his victim" en 1948, obra considerada clave para el desarrollo teórico de la Victimología como disciplina independiente. Ambos autores, señala Esbec (2000), muestran que la criminogénesis depende de una compleja relación entre víctima y victimario, en lo que Mendelshon denomina "pareja penal"; la víctima podría así contribuir, en mayor o menor medida, a su victimización, dejando de ser un sujeto pasivo. Planteamientos que continuaron desarrollando autores como Wolfgan y Ellenberg ${ }^{11}$, no sin recibir críticas de algunos grupos, por considerar que culpabilizaban a las víctimas.

El desarrollo de la psicopatología asociado al interés por las personas que sufren acontecimientos catastróficos, el estudio del trastorno de estrés postraumático, han ampliado el campo de la victimología, hacia áreas como la prevención del crimen y tratamiento a sus víctimas (Esbec, 2000).

Importante fue el desarrollo de los estudios de victimización, desarrollados en Estados Unidos, Europa y posteriormente en Chile, que dieron a conocer las formas de victimización más

"Ver "victimo-génesis" y "victim-precipitation" respectivamente. frecuentes y sus efectos en la sociedad, mostrando además la inmensa cifra negra y ayudando al mejor diseño de políticas de prevención y seguridad.

Sin duda uno de los principales logros de la víctimología, es el desarrollo de mecanismos de compensación a las víctimas del delito, manifestándose en el Primer Simposio de Victimología (Jerusalem, 1973), la necesidad de sistemas estatales de compensación a las víctimas por parte de los países, lo que fue reafirmado por la O.N.U. ${ }^{12}$ en 1985 , considerando a esta compensación como un derecho de los ciudadanos y obligación del Estado.

En Chile en tanto, y como resultado de los acuerdos del Congreso de las Naciones Unidas, se crea en 1987, el primer centro victimológico del país, el Centro de Atención a Víctimas de Atentados Sexuales (CAVAS), dependiente de la Policía de Investigaciones de Chile. Institución que se transformará en un referente en esta área tanto en Chile como Iberoamérica. Con el tiempo el equipo dirigido por Elías Escaff fue ampliando sus objetivos para, además de la asistencia integral a las víctimas de atentados sexuales, integrar la labor educativa y preventiva en la comunidad, desarrollo de investigaciones científicas, generando un modelo de intervención propio, realización de peritajes, formación de especialistas y asistencia como organismo consultor a comisiones legislativas del Congreso Nacional.

Posteriormente se continuó avanzando con la creación de distintos programas de atención a víctimas, iniciativas gubernamentales, municipales o desarrollados por ONG. En el año 1991 se crea el programa de Reparación y atención integral en salud y derechos humanos (PRAIS), dirigido a víctimas de la represión política durante el gobierno militar.

Destacan además los proyectos especializados de reparación del maltrato grave y abuso sexual del Servicio Nacional de Menores y organismos colaboradores, Centros de protección infanto-juvenil (CEPIJ) y Centros de atención integral en violencia intrafamiliar (CAIVIF) dependientes de la Fundación Opción. En el año 2001 son creados los Centros de Atención a Víc-

\footnotetext{
12 Resolución 40/43, VII Congreso de Naciones Unidas para la "Prevención del Delito y Tratamiento del delincuente" (Milán).
} 
timas de delitos violentos, dependientes del Ministerio de Justicia. En todos los casos el objetivo esencial es atención y asistencia integral (legal, psicológica y social), gratuita y especializada a niños, jóvenes, mujeres y hombres víctimas de maltrato, abuso sexual y otras situaciones de violencia.

Cabe señalar también, la prioridad programática del Plan Nacional de salud mental y psiquiatría (2000-2010) para el Ministerio de Salud, en el que se incluyen los trastornos mentales asociados a la violencia, los programas desarrollados por el Servicio nacional de la mujer, el Ministerio de Planificación y Cooperación, además de la creación de unidades policiales especializadas.

De especial relevancia es el cambio de paradigma que representa la Reforma Procesal Penal, al incorporar a la víctima del delito como un sujeto en el proceso penal, reconociendo su legitimidad para intervenir por sí mismo en el proceso. Además de lo anterior la reforma asigna al Ministerio Público el deber de protección de la víctima, labor desarrollada a través de las unidades de atención a víctimas y testigos distribuidas en todas las regiones del país.

En definitiva es posible señalar que el trabajo del psicólogo en esta área implica la intervención global en todo el proceso de victimización, abordando desde aspectos preventivos (educación y sensibilización en la comunidad), detección, evaluación y tratamiento, hasta la reducción de los efectos de victimización secundaria. Además de la participación en estudios de victimización.

\section{e. Psicología Forense}

Esta se refiere a las actividades del Psicólogo como asesor y perito de la administración de justicia, algunas ya descritas en las anteriores áreas y desempeñadas en los distintos órganos jurisdiccionales, incluyendo el trabajo del psicólogo como experto en el proceso Penal, Civil, Laboral y Contencioso-administrativo, pudiendo además agregarse su participación en los procesos Militar y Canónico.

En nuestro país, según plantea Koppmann (2002) el campo de acción habitual para las pericias psicológicas y psiquiátricas se refiere más bien al Derecho Penal y al Derecho Civil, siendo el Derecho Laboral un tópico de escaso desarrollo en cuanto a sus aspectos psicológicos forenses.
La participación del psicólogo como perito, está fundamentada en la aceptación del informe de peritos como medio de prueba en un juicio (Art. 431. Código de Procedimiento Civil).

Este informe resulta necesario y procedente según el Código de Procedimiento Civil (Art. 411) y Código de Procedimiento Penal (Art. 221) "siempre que para apreciar algún hecho o circunstancia relevante para la causa fueren necesarios o convenientes conocimientos especiales de una ciencia, arte u oficio". Informes que deberán emitirse con imparcialidad, ateniéndose a los principios de la ciencia o reglas del arte $\mathrm{u}$ oficio que profesare el perito.

La prueba pericial, como el resto de las pruebas presentadas en un juicio, será apreciada por el tribunal con total libertad, no obstante, su valoración no podrá contradecir los principios de la lógica, la experiencia y los conocimientos científicos, debiendo el tribunal fundamentar las razones que le llevaron a desestimar la prueba (Art. 297) Código de procedimiento Penal.

Las principales funciones del Psicólogo en el Proceso Civil dicen relación con la evaluación de la capacidad civil o capacidad legal, dirigida a la valoración de su estado de salud mental y en su caso, la determinación de la persona más adecuada para ostentar la tutela o curaduría del incapacitado. En casos de interdicción, señala Koppman (2002), es necesario que la enfermedad en cuestión sea grave y persistente. Otros asuntos de interés en esta área lo constituyen la impugnación de testamentos, contratos e internamientos forzosos.

También se incluyen las actuaciones de psicólogo en el ámbito del Derecho de familia, necesidad reconocida con la incorporación del psicólogo en el equipo asesor del juez en los nuevos Tribunales especializados ${ }^{13}$, cuyas labores ya fueron mencionadas en el ámbito de menor y familia.

Respecto a la intervención del psicólogo en el proceso penal, esta puede ocurrir en todas sus fases: fase de instrucción (aportando sus conocimientos en la propia investigación criminal, valoración de los testimonios, evaluación de la imputabilidad, medidas alternativas a la prisión o lesiones y secuelas psíquicas de la víctima); Fase Oral (requerido el informe psicológico sobre cualquiera de estos asuntos, como prueba

\footnotetext{
${ }^{13}$ Ley N ${ }^{\circ} 19.968$ que crea Los Tribunales de Familia.
} 
anticipada a la vista); Fase de ejecución de sentencia (en asuntos de vigilancia penitenciaria, evaluación de peligrosidad del interno, concesión de permisos penitenciarios o sobre la conveniencia de abandonar la prisión a causa de trastornos mentales después de sentencia firme (Esbec, 2000).

Sin duda uno de los peritajes que revisten más interés en esta área dice relación con el concepto de imputabilidad, imputar significa atribuir el hecho al conocimiento y la libre voluntad del agente (Esbec, 2000). Siendo una condición necesaria a efectos de responsabilidad penal, que "el individuo debe poseer salud mental y madurez psíquica en grado suficiente, o que en el momento de cometer el hecho que se le imputa, haya estado en plena posesión de sus facultades mentales" (Koppman, 2002).

El Código Penal (Art. 10-12) establece las circunstancias que afectan a la imputabilidad. Existiendo tres posibles situaciones médico-legales en la cuestión de la imputabilidad en las personas con trastornos psíquicos: inimputable, atenuación de la imputabilidad e imputable. En Chile los jueces solicitan que la pericia penal contenga expresamente un pronunciamiento sobre la imputabilidad del procesado, por lo que resulta fundamental considerar la existencia o no de algún desorden mental y la eventual participación que esa alteración tiene en el compromiso de la voluntad o de la inteligencia (op. cit.).

Otro tema de interés para los Psicólogos es la valoración del discernimiento ${ }^{14}$, en razón de lo que plantea el Código Penal (Art. 10 inciso 3), que establece que: está exento de responsabilidad penal "El mayor de dieciséis años y menor de dieciocho, a no ser que conste que ha obrado con discernimiento". Esta condición según Koppman (2002) es homologable a un estado de desarrollo que implica madurez biológica y mental para poder distinguir el bien del mal, o la ilicitud de un hecho punitivo ante la ley. El individuo debe estar consciente del mundo externo, razonar y juzgar sobre lo moralmente correcto y ser capaz de controlarse.

La valoración de la peligrosidad, se basa en la necesidad de protección de la sociedad, ante quienes puedan poner en riesgo la integridad individual o colectiva, e implica determinar la probabilidad de que el sujeto pueda cometer

it Este trámite es suprimido en el nuevo proyecto de Ley sobre Responsabilidad Penal Juvenil. actos delictivos futuros. Si bien se han elaborado instrumentos que intentan ayudar en su valoración, según Esbec (2000) resulta ingenuo pensar que la peligrosidad pueda reducirse a una dimensión o producto de varias dimensiones; lo imprevisible de la conducta humana y la debilidad de los criterios científicos aplicados, hacen necesario perfeccionar aún más los sistemas de evaluación y evitar así lamentables consecuencias.

La labor del psicólogo forense es transversal a todas las áreas, pudiendo ser realizada por profesionales de instituciones públicas o bien del ámbito privado. No obstante la Ley da preferencia a los servicios públicos. En nuestro país históricamente la institución encargada de asistir a los jueces en en pericias médico-forenses es el Servicio Médico Legal, en el que se desempeñan psicólogos en el área de salud mental, el Cavas también realiza informes de este tipo desde principios de la década de los noventa, a los que se suman los psicólogos que trabajan en el ámbito de menores, penitenciario, etc. Cabe señalar que actualmente no existe criterios comunes y estandarizados en el desarrollo de este trabajo por parte de las distintas instituciones, por lo que actualmente se están haciendo esfuerzos por compartir información y establecer una mejor coordinación entre los profesionales que trabajan en ellas.

\section{f. Area de Docencia e Investigación}

En nuestro país, sólo algunas de las carreras de Psicología de las diversas Universidades existentes, tanto públicas como privadas, contemplan en los últimos años alguna asignatura relacionada con la Psicología Jurídica, correspondiendo habitualmente a cursos de tipo electivo.

Si bien en el último tiempo se han intensificado las investigaciones, especialmente en algunas áreas de la Psicología Jurídica (ámbito penitenciario, agresores y víctimas de maltrato y abuso sexual), llevadas a cabo principalmente por Universidades, instituciones públicas u Organismos no Gubernamentales (ONG), éstas aún resultan insuficientes, situación que se relaciona con la escasa oferta de formación de Postgrado en el área orientada a la producción de investigación y conocimiento en el tema. Si bien han surgido varios programas y cursos de Postítulo, estos buscan desarrollar competencias esfecíficas para la intervención en el área. 
Hitos importantes en el desarrollo de la disciplina en nuestro país, lo constituyen la organización del Primer y Quinto Congreso Iberoamericano de Psicología Jurídica, realizados en el año 1995 y 2003 respectivamente, los cuales contaron con el patrocinio de la correspondiente asociación, creada en el año 1993, con actual presidencia en Chile. Destacada también la reciente creación de la Asociación Chilena de Psicología Jurídica y la realización de su Primer Encuentro Nacional, en agosto del año 2004, instancia que busca vincular a los psicólogos que se desempeñan en esta área y promover el desarrollo de la misma.

\section{Comentarios Finales}

Las diferentes transformaciones políticas, sociales y científicas, hacen emerger nuevos paradigmas y disciplinas, así la Psicología Jurídica en la que se entrecruzan distintos saberes y discursos, nos exige la reflexión y el análisis, motivados por la complejidad de los problemas que la ocupan.

La Psicología Jurídica, a primera vista se nos aparece más bien definida por lo que hace, que por lo que sabe, caracterizándose por su carácter profesional e interventivo, quedando supeditadas epistémica, teórica y metodológicamente al conjunto de la Psicología y el Derecho. Quizá este fenómeno responda a que el Derecho y su visión positivista de la ley, demanda a la Psicología un pronunciamiento por el sujeto en términos de categorías y sistemas conceptuales. Esto nos desafia a rescatar en nuestro trabajo la subjetividad del individuo salvando la doble determinación y distinción tensional entre sujeto y sociedad (orden legal).

La específica dimensión en la que se articulan las prácticas de la Psicología Jurídica, determina y posibilita su intervención, en este sentido Clemente (1997) plantea que en pocos lugares las conductas, cogniciones y experiencias de las personas dependen tanto del lugar donde se producen como en el terreno de lo jurídico, siendo imposible estudiar ese comportamiento abstrayéndose de esa realidad. Así, la existencia de la
Psicología Jurídica pasa por estar inserta en un ambiente creado por la organización jurídica, que dista de otras realidades vivenciadas fuera de este ámbito específico.

La Psicología jurídica debe interpretar esta complejidad, entendiendo a la teoría como la posibilidad de iluminar las contradicciones (Adorno, 1973). De estas consideraciones derivan opciones específicas respecto de su configuración teórica, objeto de estudio, metodología y las prácticas que la constituyen. Y requieren la revisión permanente de sus fines y herramientas utilizadas.

En este sentido, la pregunta acerca del quehacer del psicólogo en este ámbito y en cada una de sus áreas resulta relevante, su respuesta ha de servir para orientar su labor, considerando no sólo la inclusión de aspectos técnicos, sino también de carácter ético, evitando así perderse en el sistema y constituirse sólo en un "engranaje más". De esta manera, como señala Urra (2003), sería urgente actuar de acuerdo a la ciencia y a la conciencia, protegiendo la dignidad de la persona humana y los derechos inviolables que son inherentes a ésta.

Finalmente es importante recordar que el desarrollo de la Psicología Jurídica en el país se encuentra en ciernes, no siendo posible aún apreciar totalmente el impacto de ésta en la administración de justicia. No hay duda de que su desarrollo representa un desafío importante para la colectividad de profesionales de la Psicología en nuestro país, resultando importante destacar la necesidad de crear mecanismos que no tan sólo validen la calificación técnica para el ejercicio de la especialidad, sino que también garanticen la probidad de los profesionales que se dedican a esta delicada tarea, ya que sólo en la medida en que seamos capaces de demostrar que el aporte de la Psicología Jurídica resulta interesante y útil, este podrá ser validado, abriendo nuevos espacios de desarrollo para la misma, en el entendido de que la razón final de su intervención se refiere a proporcionar una mejor administración de justicia para todos los sujetos involucrados en ella y la sociedad en su conjunto. 


\section{PSICOLOGÍA Y LEY: "UNA RELACIÓN PARTICULAR"}

\section{Referencias}

Asociación Iberoamericana de Psicología Jurídica (1995). Anales del Ier Congreso Iberoamericano de Psicología Juridica. Santiago- Chile.

Bajet, J. (2003). La Psicología Juridica: Pasado y presente de su breve historia. Centro de estudios jurídicos del Colegio de Psicólogos de España.

Blair, K. (1972). Psychology and law. American Psychologist. Vol 27 (5): 499-503.

Castex, M. (1996). ¿Qué significa la Psicologia forensc? Publicación interna de la Cátedra de Psicología Forense de la Facultad de Psicología de la Universidad de Buenos Aires, Argentina.

Clemente, M. (1997). Fundamentos de la Psicología Juridica. Madrid. Pirámide.

Clemente, M. \& y Núñez, J. (1997). "La organización social informal en la prisión", en "Psicología Jurídica penitenciaria". Madrid. España. Fundación UniversidadEmpresa.

Esbec, Enrique (2000). Psicología Forense y Tratamiento Jurídico-legal de la discapacidad. España. Edisofer.

Escaff, E. (2000). Psicología Juridica en Chile. Trabajo presentado en el I Congreso Hispano-Alemán de Psicología Juridica.

Fabian, Thomas (2000). Legal Psychology in Germany, Anuario de Psicología Jurídica, págs. 157-171.

Garzón, A. (1989). Dimensiones Psicológicas y sociales de la decisión Judicial. Psicología y Justicia. Valencia. España. Promolibro.

Kapardis, A. (1997). Psychology and law. A critical introduction. United Kingdom. Cambridge University Press.

Koppmann, A., Portilla, D. (2002). La actuación del psicólogo forense en Santiago de Chile. España. Infocop.

Loh, W. D. (1981). Perspectives in psychology and the law. Journal of Applied Social Psychology. 11, 314-355.
Mira, E. (1961). Manual de psicología jurídica: Barcclona. Salvat.

Mohanan, J. \& Loftus (1982). The psychology of law. Annual Review of Psychology, Vol. 33: 441-475.

Muñoz Sabaté, L. \& Bayćs, R. (1980). Introducción a la Psicología Jurídica. México. Trillas.

Muñoz Sabaté, L. (1975). Métodos y clementos para una Psicología Jurídica. Amuario de Sociología y Psicología Juridica. No 2. Madrid.

Paleari, A. (2002). El psicólogo del Servicio Penitenciario yel psicodiagnóstico. Revista de Estudios Criminológicos y Penitenciarios. No 5. Santiago de Chilc.

Retamal, S. (2000). El Quć-Hacer del Psicólogo en cl Sistema Penitenciario. Revista de Estudios Criminológicos y Penitenciarios. No 1. Santiago de Chile.

Ruiz, J.I. \& Páez, D. (2001). Satisfacción laboral, burnout y clima emocional en empleados de prisiones: Un estudio exploratorio. Acta colombiana de Psicología. Monográfico sobre Psicología Jurídica.

Sobral, J. \& Prieto, A. (1994). Psicología y Ley. Madrid: Eudema.

Sobral, J.; Arce, R. \& Prieto, A. (1994). Manual de Psicología Jurídica. España: Paidós.

Soria, M. (1998). Psicología y práctica juridica. Barcelona: Ed. Ariel.

Soria, M. A. (1993). La victima: entre la justicia y la delincuencia. Aspectos Psicológicos, Sociales y Juridicos de la Victimización. Barcelona. España. P.P.U.

Tapp, J. (1976). Psychology and the law: An overture. Annual Review of Psychology. Vol. 27 35: 339-404.

Teixido, R. (1978). Una aproximación filosófica a la Psicología Jurídica. Anuario de Psicologia y Sociología Jurídica. No 5 .

Urra, J. (2003). Agresor Serual. Casos Reales, Riesgo de Reincidencia. E.O.S. 